${ }^{3}$ R. A. Fisher \& F. YATEs, Statistical Tables for Biological Agriculture and Medical Research. 3rd edition, London, 1948, Table XXII.

4 GEORGE W. REITWIESNER, "An ENIAC determination of $\pi$ and $e$ to more than 2000 decimal places," $M T A C$, v. 4, 1950, p. 11-15.

s N. C. Metropolis, G. Reitwiesner, \& J. von Neumann, "Statistical treatment of values of first 2000 decimal digits of $e$ and $\pi$ calculated on the ENIAC," MTAC, v. 4, 1950, p. 109-111.

${ }^{\circ}$ W. Feller, Probability Theory and Its Applications. Volume 1, New York, 1950, p. 175-181.

${ }^{7} \mathrm{~F}$. GRUENBERGER, "Further statistics on the digits of $e, " M T A C, \mathrm{v} .6,1952, \mathrm{p}$. 123-134.

\title{
A Method for the Evaluation of a System of Boolean Algebraic Equations
}

With the advent of large scale electronic devices whose logical design is described by a system of Boolean algebraic equations, a method to mechanize the evaluation of such a system and shorten this evaluation with respect to time will be increasingly useful. Such a method will be described in this paper.

The problem may be described as follows: Given a set of $n$ variables, $Q^{k}$, $(k=1,2, \cdots, n)$ each of which may take on the value 1 (true) or 0 (false) at any time $t$; then the value of any $Q^{k}$ at time $t+1$ may be defined by the system of Boolean equations

$$
\begin{aligned}
R_{t}{ }^{k} & =f_{k}\left(Q_{t}{ }^{q}\right) \\
S_{t}{ }^{k} & =g_{k}\left(Q_{t^{q}}\right) \\
Q^{k}{ }_{t+1} & =\phi\left(Q_{t^{k}}{ }^{2} R_{t^{k}}{ }^{k} S_{t}{ }^{k}\right)
\end{aligned}
$$

where $1 \leq q \leq n$. For example, the recirculation loop of a dynamic flip-flop may be defined simply by

$$
Q^{k}{ }_{t+1}=\phi\left(Q_{t}{ }^{k}, R_{t}{ }^{k}, S_{t}{ }^{k}\right)=R_{t}^{k} .
$$

In another system, a more complex definition

$$
Q^{k}{ }_{t+1}=\phi\left(Q_{t}{ }^{k}, R_{t}{ }^{k}, S_{t}{ }^{k}\right)=Q_{t}{ }^{k} \cdot \overline{R_{t}{ }^{k}} \cdot \overline{S_{t}{ }^{k}}+R_{t}{ }^{k} \cdot \overline{S_{t}{ }^{k}}+\overline{Q_{t}{ }^{k}} \cdot R_{t}{ }^{k} \cdot S_{t}{ }^{k}
$$

may be taken, where $R_{t}{ }^{k}$ and $S_{t}{ }^{k}$ are the two inputs to flip-flop $Q^{k}$.

We shall use the symbols for conjunction, disjunction, and negation

$$
\begin{array}{lll}
Q^{1} \cdot Q^{2} & \text { " } Q \text { and } Q^{2 "} & \text { conjunction } \\
Q^{1}+Q^{2} & \text { " } Q \text { or } Q^{2 "} & \text { disjunction } \\
Q^{1} & \text { "Not } Q 1 " & \text { negation }
\end{array}
$$

which are defined by the truth tables ${ }^{1}$

$\begin{array}{ccccc}Q^{1} & Q^{2} & Q^{1} \cdot Q^{2} & Q^{1}+Q^{2} & \overline{Q^{1}} \\ 0 & 0 & 0 & 0 & 1 \\ 0 & 1 & 0 & 1 & 1 \\ 1 & 0 & 0 & 1 & 0 \\ 1 & 1 & 1 & 1 & 0 .\end{array}$

A "term" is defined as one or more variables conjoined together, e.g., $Q^{1} \cdot Q^{2} \cdot \overline{Q^{3}}$; and an "equation" as $M$ terms, $T_{m},(m=1,2, \cdots, M)$ disjoined together, e.g., $Q^{1} \cdot Q^{2} \cdot \overline{Q^{3}}+Q^{1} \cdot Q^{4}$. Now note that the value of a term is zero if any variable in 
that term has the value zero, and the value of an equation is one if any term in that equation has the value one. We will deal only with the case where all of the expressions on the right are in normal disjunctive form, but this represents no restriction on the method since any equation in the above form may be so written.

Our method for solving this system of equations involves the use of punched cards. While this description is in terms of IBM punched cards, the method is, of course, not restricted to this type of card. Cards and a key punch are the only tools required. In fact, the key punch is required only to initially punch the cards as described below, and is not used thereafter in the actual process of evaluation.

Two types of cards must be defined and punched in order to implement the evaluation. Cards of type I each represent the $m^{\text {th }}$ term of the equation for $R^{k}$ or $S^{k}$ and are labeled " $R^{k} T_{m}$ " or " $S^{k} T_{m}$ "; these are the "equation" cards. Cards of type II represent the value of $Q^{k}(1$ or 0$)$ and are labeled $Q^{k} T$ or $Q^{k} F$, respectively; these are the "value" cards and one or the other is selected for use at time $t$ for each $k$. The problem may now be restated as: given the set of cards of type II containing the appropriate card $Q^{k} T$ or $Q^{k} F$ for all $Q^{k}$ at time $t$, and further given the equations as represented by the deck of cards of type I, construct a set of cards of type II representing the value of each $Q^{k}$ at time $t+1$.

The twelve rows and 80 columns of the IBM card may be thought of as a single pair of rows and 480 "columns." Let this column number correspond to $k$ and let us represent variable $Q^{k}$ by one of three possible combinations of punches or no punches in the pair of rows of column $k$.

Thus cards of type I are constructed such that the card $R^{k} T_{m}$ has column $q$ punched as follows:

i) a punch in row 1 if $Q^{q}$ is a variable in term $m$ of the equation for $R^{k}$,

ii) a punch in row 2 if $\overline{Q^{a}}$ is a variable in term $m$ of the equation for $R^{k}$, or,

iii) no punch in column $q$ if neither $Q^{q}$ nor $\overline{Q^{q}}$ appears as a variable in term $m$ of the equation for $R^{k}$.

The card $S^{k} T_{m}$ is constructed in an entirely similar way.

Cards of type II are constructed in a manner which is somewhat analogous to the above. The same 480 columns of a single pair of rows is considered. Now, however, there is one and only one punch in column $k$, and all rows of all other columns are punched; i.e.,

i) the punch in column $k$ is in row 1 if $Q^{k}$ is true . . . in this case the card is labeled $Q^{k} T$;

ii) the punch in column $k$ is in row 2 if $Q^{k}$ is false $\left(\overline{Q^{k}}\right.$ is true) . . in this case the card is labeled $Q^{k} F$;

iii) both rows of all other columns are punched.

A "deck" of cards of type II consists of all of the cards $Q^{k} T$ and $Q^{k} F$. A "set" of these cards at time $t$ consists of one and only one card $Q^{k} T$ or $Q^{k} F$ representing the value of each $Q^{k}$ at time $t$. Thus there are twice as many cards in a deck of type II as in a set of type II. It will be convenient to have two decks of type II so that one set of cards may be made up for time $t$ from one deck and, using this set and the entire deck of cards of type I, a set of cards of type II for time $t+1$ may be compiled from the other deck. 
We shall call the set of cards of type II, for time $t$, the "this time table" and those for time $t+1$ the "next time table." Note that after we have formed the complete next time table, we may redefine it as the new this time table and proceed to form a new next time table for time $t+2$ from the (now) unused deck of type II. This process may continue as long as required, and is essentially that of evaluation of the equations.

The process is carried out as follows: The this time table is stacked and remains undisturbed (but not unused) until the next time table is completed. The entire this time table is physically aligned so that each position on the card representing each row and column is directly in line with the corresponding position of all other cards in the set. With these cards so arranged, it is easy to determine which positions are punched in every card of the set; this is most simply done visually by simply sighting through the entire deck. Further, we may now observe that we can sight through one and only one of the two rows in every column. That this is the this time table for time $t$ has the significance that if we can sight thru row 1 of column $k$, then $Q_{t}{ }^{k}=1$, or, if we can sight thru row 2 of column $k$, then $Q_{t}{ }^{k}=0$. Thus by simply aligning the cards of the this time table, we can read the values of $Q_{t}{ }^{k}$ required to evaluate $\phi$ (Eq. 3).

We may now evaluate $R_{t}{ }^{k}$ (Eq. 1) by the following measures if the cards $R^{k} T_{m}$ of type $\mathrm{I}$ are arranged in ascending order of $k$ and $m$ : Place card $R^{1} T_{1}$ in alignment with the this time table as above. If we can sight thru the deck of cards thus formed wherever $R^{1} T_{1}$ is punched, then $R_{t}{ }^{1}=1$ and it is not necessary to examine cards $R^{1} T_{2}, R^{1} T_{3}, \cdots, R^{1} T_{M}$, at this time. If not, we remove $R^{1} T_{1}$ and proceed to examine $R^{1} T_{2}$ in the same way. This process continues until either 1) we find $R^{1} T_{m}$ satisfying the condition that we can sight thru the deck consisting of it and the this time table, in which case $R_{t}{ }^{1}=1$, or 2) we have examined all $R^{1} T_{m}$ without satisfying the condition, in which case $R_{t}{ }^{1}=0$. Having thus evaluated $R_{t}{ }^{1}$, we proceed in the same manner to evaluate $S_{t}{ }^{1}$. When this is done, we are now in a position to solve for $Q^{1}{ }_{t+1}$ from equation 3 . In exactly the same way we proceed to evaluate in order $R_{t}{ }^{2}, S_{t}{ }^{2}, Q^{2}{ }_{t+1}, R_{t}{ }^{3}, S_{t}{ }^{3}, Q^{3}{ }_{t+1}, \cdots, R_{t}{ }^{n}, S_{t}{ }^{n}, Q^{n}{ }_{t+1}$. The values of the $Q$ 's are recorded as the next time table by forming the proper set from the available deck of cards of type II. The values of $R$ and $S$ need not be retained.

The above description in which IBM cards are employed requires that $N \leq 480$. It is easy to see that this number may be increased by increasing the number of cards of type I and type II appropriately. However, the more usual problem is to reduce the number of cards employed when $n$ is sufficiently less than 480 , this being the more usual case.

The number of cards of type II employed is perfectly straightforward: If $n \leq 480$, then the number in a deck is $2 n$ and that in a set is $n$; if $480<n \leq 960$, then the number in a deck is $4 n$ and that in a set is $2 n$; and so forth. But if $n$ is sufficiently small, e.g., $n=240$, then we may make certain types of combinations of the equation cards (type I). For example, we may represent $R^{k} T_{m}$ and $S^{k} T_{m}$ on the same card, or we may represent two terms of $R^{k}$ on one card. Such combinations of cards serve to reduce the time required to carry out the evaluation, where $n$ is sufficiently small, without loss of generalization. They require certain 
obvious modifications of the configuration of the value cards (type II) in the form of duplication of information.

When the problem actually is solved as described above, it is convenient but not necessary to have such devices as a box of some transparent material in which to stack the this time table, perhaps with a strategically placed light source to assist in observations. In fact, the design has been completed for a device to completely mechanize the process described so that the human operator has only to feed cards to a card reader with the solution carried out and the results recorded automatically.

\section{J. A. Postley}

Hughes Research and Development Laboratories

Culver City, California

${ }^{1}$ Hans Reichenbach, Elements of Symbolic Logic. The Macmillan Company, New York, 1947. The notation used in this paper is that of Reichenbach except that his symbol " $v$ " is replaced by the symbol "+".

2 E. C. Nelson, "An algebraic theory for use in digital computer design," Transactions of the IRE Professional Group on Electronic Computers, vol. EC-3, p. 12-21, September 1954.

${ }^{3}$ Engineering Research Associates, High-Speed Computing Devices. McGraw-Hill Book Company, New York, 1950.

4 Arthur W. Burks, Don W. Warren, \& Jesse B. Wright, "An analysis of a logical machine using parenthesis-free notation," $M T A C$, v. 8, 1954, p. 53-57.

\section{Tables for the Determination of Fundamental Solutions of Equations in the Theory of Compressible Fluids}

1. The pseudo-logarithmic plane. It has been shown $n^{1,2,3}$ that it is useful, when applying the hodograph method, ${ }^{4}$ to consider the stream function $\psi$ and the potential function $\phi$ of compressible fluids in the so-called pseudo-logarithmic plane. In the case when the pressure density equation is $p=\sigma \rho^{\gamma}, \sigma, \gamma$ being constants, and for subsonic flows, the cartesian coordinates of the pseudo-logarithmic plane are

$$
\lambda=\frac{1}{2} \log \left[\frac{1-T}{1+T}\left(\frac{1+h T}{1-h T}\right)^{1 / h}\right], \quad T=\left(1-M^{2}\right)^{\frac{1}{2}}, \quad h=\left(\frac{\gamma-1}{\gamma+1}\right)^{\frac{1}{2}},
$$

and $\theta$ where $M$ is the Mach number and $\theta$ is the angle which the velocity vector forms with the positive $x$ direction of the physical plane (i.e., the plane in which the flow actually takes place). ${ }^{5}$

The equations for the potential $(\phi)$ and the stream function $(\psi)$ assume, when considering the flow in the $\lambda, \theta$-plane, the form

$$
\begin{gathered}
\phi_{\lambda \lambda}+\phi_{\theta \theta}+\left[l^{l}\left(l^{-\frac{1}{2}}\right)_{\lambda}\right] \phi_{\lambda}=0, \quad \phi_{\lambda} \equiv \frac{\partial \phi}{\partial \lambda}, \cdots \\
\psi_{\lambda \lambda}+\psi_{\theta \theta}+\left[l^{-\frac{1}{2}}\left(l^{\frac{1}{2}}\right)_{\lambda}\right] \psi_{\lambda}=0 .
\end{gathered}
$$

